\title{
Modeling and Detection of Wrinkles in Aging Human Faces Using Marked Point Processes
}

\author{
Nazre Batool` and Rama Chellappa \\ Department of Electrical and Computer Engineering and the Center \\ for Automation Research, UMIACS, University of Maryland, \\ College Park, MD 20742, USA
}

\begin{abstract}
In this paper we propose a new generative model for wrinkles on aging human faces using Marked Point Processes (MPP). Wrinkles are considered as stochastic spatial arrangements of sequences of line segments, and detected in an image by proper localization of line segments. The intensity gradients are used to detect more probable locations and a prior probability model is used to constrain properties of line segments. Wrinkles are localized by sampling MPP using the Reversible Jump Markov Chain Monte Carlo (RJMCMC) algorithm. We also present an evaluation setup to measure the performance of the proposed model. We present results on a variety of images obtained from the Internet to illustrate the performance of the proposed model.
\end{abstract}

Keywords: Modeling of wrinkles, Markov Point Process, Reversible Jump Markov Chain Monte Carlo, stochastic geometrical model.

\section{Introduction}

Wrinkles and fine lines are important facial features present in most aging faces. An accurate image-based analysis of these features can play an important role in relevant aging applications. Much of the work done in aging applications, e.g. age estimation and simulation, is based on the incorporation of wrinkles as texture features [12]3. A few attempts have been made to evaluate wrinkles as edges [45]. However, image-based detection and analysis of wrinkles has mostly remained unaddressed. This can probably be attributed to the nature of the artifacts associated with wrinkles as well as the challenges posed by drastically varying skin appearance in images with variations in illumination and acquisition angles [6]. In this paper, we propose a model to detect and analyze wrinkles explicitly. The basis for our methodology is based on the following observations. First, wrinkles create the appearance of texture on skin, but at high resolution, wrinkles do not depict any repetitive/homogeneous pattern. Second, wrinkles cannot be categorized as boundaries between multiple textures. Third, a wrinkle is usually a discontinuity

\footnotetext{
* The first author would like to acknowledge the support of Fulbright/HEC(Pakistan)/USAID PhD Scholarship. However, the contents of this publication have not been approved by the respresenting agencies/governments of the USA/Pakistan.
}

A. Fusiello et al. (Eds.): ECCV 2012 Ws/Demos, Part II, LNCS 7584, pp. 178-188, 2012.

(C) Springer-Verlag Berlin Heidelberg 2012 


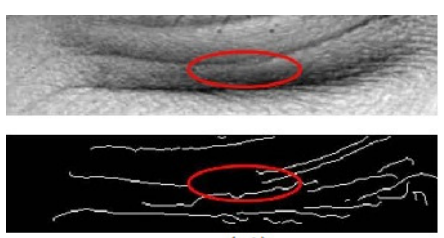

(a1)

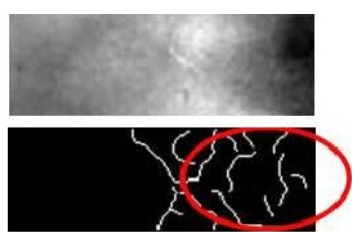

(a2)



(c1)

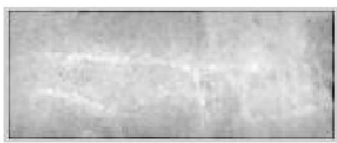

(c2)

(b1)

(b2)

(b3)

(b4)

Fig. 1. (Left) Results with the Canny edge detector - (a1) Wrinkles as broken edges (a2) False edges due to illumination (b1) threshold $=0.3$ (b2) threshold $=0.2$ (b3) threshold $=0.1$ (b4) wrinkles detected as line segments (Right) LoG filtering (c1) Original Image (c2) Filtered image with $\sigma=0.5$

in background inhomogeneous skin texture. And finally, wrinkles do not always appear as continuous edges.

It can be argued that an accurate edge detector can detect wrinkles. We present some typical results using the Canny edge detector in Figure 1, In Figure 1(a1) the encircled edge is disconnected despite the original wrinkle being continuous. In Figure 1(a2) illumination discontinuities are marked as edges. Figure 1(b1-b3) show the erroneous edges if the threshold of edge detector is set too low. To explain our motivation, Figure 1(b4) shows the results of our model for comparison. A lower number of erroneous edges as well as conitnutity of edges representing detected wrinkles can be observed. In short, although intensity gradients are well picked up as edges by an edge detector, no distinction is made between intensity gradients due to wrinkles, illumination discontinuities or other factors. A method capable of filtering intensity gradients based on the probability of their being wrinkles is needed. We propose to incorporate prior semantic information available from typical appearances of wrinkles on human faces for that purpose. Wrinkles are modeled as a stochastic spatial process of 'line segments' in a Bayesian framework using Marked Point Process (MPP) 7 . In terms of methodology, our work is related to the work by Soitca et al. 8] for detection of road networks in satellite images. The main contribution of our work is the modeling of wrinkles as a spatial line process to incorporate prior information about wrinkles and detection of wrinkles explicitly as line segments. Also, as compared to the work reported in [4]5, we experiment with a larger variety of images obtained from the Internet.

\section{Marked Point Process (MPP)}

A spatial point process is a random process of occurrence of points in space defined with respect to a Poisson measure. Normally a set of random parameters 



Fig. 2. (a)Attraction interaction creates doubly connected segment (b) Rejection interaction

is also associated with a point, called mark, describing its specific properties. In this work, the marked points represent line segments. Two segments can interact with each other due to spatial proximity in the image plane. An attraction interaction results in interconnection of line segments whereas a rejection interaction prevents overlappping or congested parallel segments. A resulting sequence of interconnected line segments represents a wrinkle. An MPP model consists of a prior probability model and a data likelihood term. The prior model imposes geometric constraints on the spatial arrangements of line segments whereas the data likelihood determines the probability of occurrence of line segments depending on the intensity gradients at image sites. A marked point is given by $w_{i}=\left(s_{i}, m_{i}\right)$ where $s_{i}=\left(x_{i}, y_{i}\right) \in S \subset \mathbb{R}^{2}$ is the location of center of the segment and $m_{i}=\left(l_{i}, \theta_{i}\right) \in M$ is the mark consisting of two parameters denoting the length and orientation of the segment, respectively. The continuous space for parameters is given by $M=\left[l_{\min }, l_{\max }\right] \times\left[\theta_{\min }, \theta_{\max }\right]$. The line segments $\left\{\mathbf{w}=w_{i}, i=1, \ldots, n\right\}$ can be considered as a realization of the MPP on the space $S \times M$. For further details on MPP, the interested reader is referred to [7]. The probability density of MPP can be represented by the Gibbs distribution:

$$
f(\mathbf{w})=c \beta^{n(\mathbf{w})} \exp (-U(\mathbf{w})) \text { where } U(\mathbf{w})=U_{P}(\mathbf{w})+U_{D}(\mathbf{w}) .
$$

The term $c$ is the normalizing constant, $\beta$ is the intensity of the point process, $n(\mathbf{w})$ and $U(\mathbf{w})$ are the number of line segments and the total energy of the realization $\mathbf{w}$, respectively. The terms $U_{P}(\mathbf{w})$ and $U_{D}(\mathbf{w})$ correspond to the energies contributed by the prior model and the data likelihood term, respectively.

\subsection{Prior Model}

The prior model determines more likely geometric properties of the line segments and spatial interactions between neighboring line segments. The parameters $\left\{l_{i}, \theta_{i}\right\}$ are sampled from a uniform distribution where the parameters of the uniform distribution set limits on the geometric properties of the line segments:

$$
l_{i} \sim \mathcal{U}\left(\left[l_{\min }, l_{\max }\right]\right) \text { and } \theta_{i} \sim \mathcal{U}\left(\left[\theta_{\min }, \theta_{\max }\right]\right) .
$$

Under the prior model, two segments can interact with each other in spatial domain i.e. the segments can influence each other's probability of being included in a realization of the MPP due to their spatial proximity. We propose a rejection 
interaction which penalizes the birth (addition) of overlapping or congested segments. Let the rejection interaction between two segments $\left\{w_{i}, w_{j}\right\}$ be denoted by $w_{i} \stackrel{r}{\sim} w_{j}$. A rectangular rejection region of a certain width exists around each existing segment in the configuration. Figure 9(b) shows the rectangular rejection interaction region around an existing segment $\mathrm{w}_{1}$. The new segment is penalized if it overlaps with the rejection region of any existing segment. The rejection interaction is modeled through the term $q\left(w_{i}, w_{j}\right)$ as follows. The parameter $\gamma$ determines the penalty assigned to the interacting new segment.

$$
q\left(w_{i}, w_{j}\right)=\gamma \times \mathbb{I}_{\left(w_{i} \stackrel{r}{\sim} w_{j}\right)} \text { where } \mathbb{I}_{\left(w_{i} \stackrel{r}{\sim} w_{j}\right)}=\left\{\begin{array}{l}
1 \text { if } w_{i} \stackrel{r}{\sim} w_{j} \\
0 \text { if } w_{i} \stackrel{\sim}{\sim} w_{j}
\end{array}\right.
$$

The total energy for the prior model is given by:

$$
U_{P}(\mathbf{w})=\sum_{\substack{\left(w_{i}, w_{j}\right) \in \mathbf{w} \\ w_{i} \stackrel{\sim}{\sim} w_{j}}} q\left(w_{i}, w_{j}\right)
$$

\subsection{Data Likelihood Energy}

The data likelihood determines the probability of occurrence of line segments at image sites. An image is filtered with a Laplacian of Gaussian (LoG) filter to highlight the intensity gradients caused by wrinkles. Figure 1(c1, c2) shows the LoG filter response to a low resolution forehead image. We selected the standard deviation $(\sigma=0.5)$ for LoG filters. Let $D_{i}$ denote the pixels corresponding to the segment $w_{i}$ and $d_{\text {min }}$ denote the minimum filter response observed in the given image. Then the likelihood energy for the segment $w_{i}$ is given by

$$
U_{D}\left(w_{i}\right)=-\left[\alpha \sum_{d \in D_{i}}\left(d-d_{\min }\right)^{2}\right]
$$

\section{Simulation of the Marked Point Process}

For an MPP, the number of objects is not known a priori and the MetropolisHastings algorithm cannot be used to sample from distributions. Green [9] presented the Reversible Jump Markov Chain Monte Carlo (RJMCMC) algorithm to allow configurations of different dimensions, i.e. different numbers of line segments. The algorithm allows jumps between states in configurations of different dimensions. Given that the state $\mathbf{w}$ is changed to $\mathbf{w}^{\prime}$, the algorithm requires the matching of dimensions for two configurations. This is achieved by using an auxiliary random variable $\omega$, sampled from an arbitrary distribution $g(\omega)$, and a bijective transformation $\mathcal{T}$ such that $\mathbf{w}^{\prime}=\mathcal{T}(\mathbf{w}, \omega)$. The acceptance probability $\phi$ of a jump modifying configuration $\mathbf{w}$ to the configuration $\mathbf{w}^{\prime}$ is then modified by the Jacobian of the transformation, $\left|\frac{\partial \mathcal{T}}{\partial(\mathbf{w}, \omega)}\right|$, as follows.

$$
\phi\left(\mathbf{w} \rightarrow \mathbf{w}^{\prime}\right)=\min \{1, R\} \text { where } R=\frac{f\left(\mathbf{w}^{\prime}\right)}{f(\mathbf{w}) g(\omega)}\left|\frac{\partial \mathcal{T}}{\partial(\mathbf{w}, \omega)}\right| \times \frac{\operatorname{prob}(\text { reverse jump })}{\operatorname{prob}(\text { forward jump })}
$$




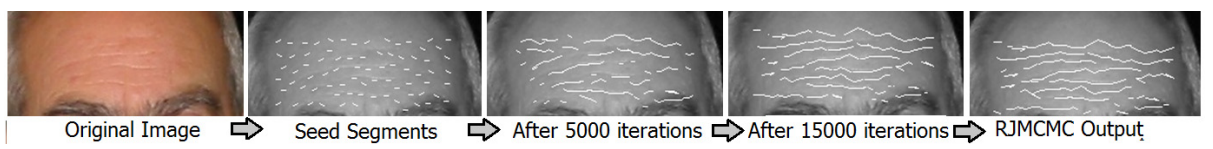

Fig. 3. Step-by-step process for the detection of wrinkles in an image

The term $R$ is called the acceptance ratio and needs to be calculated for every move under RJMCMC. Furthermore, simulated annealing is used to reach the global minimum of the Gibbs energy $U(\mathbf{w})$ in equation (11) by replacing the probability density $f(\mathbf{w})$ by $f(\mathbf{w})^{\frac{1}{T}}$ in RJMCMC algorithm. The cooling schedule for $T$ is given by $T=\frac{T_{0}}{\log (1+i)}$ where $i$ denotes the current iteration and $T_{0}$ is selected to be 10 . The simulation of point processes involves birth and death moves, where points are either added to or deleted from the configuration, changing its dimension. The reverse jumps (death) ensure the reversibility of the Markov chain (See Geyer [10] for a proof and further discussion).

\subsection{Birth and Death of Singly/Doubly Connected Segments}

A new segment is sampled from the distribution $g(\omega)$ and connected to a randomly selected segment from the configuration with at least one free end. This move can add a singly or doubly connected segment based on spatial interactions among segments. Figure 2(a) shows an example of birth of a doubly connected segment as a result of attraction interaction. The left end of the existing segment $\mathrm{w}_{2}$ is selected to be connected to the new segment. An attraction interaction region is defined to be a conic region connected to that end point. An existing segment in that region ( $w_{1}$ in this case) results in the new segment being connected at both sides between the two existing segments. A segment is not allowed to be connected to more than one segment at either end point. Let $\left\{n_{c 0}, n_{c 1}, n_{c 2}\right\}$ be the number of line segments with both ends free, connected at one end and connected at both ends respectively. Let $\left(x_{e}, y_{e}\right)$ be the coordinates of a free end point selected randomly out of $2 n_{c 0}+n_{c 1}$ available free end points in the current configuration. Let us assume that this is going to be a singly connected segment and let $\omega=\left(\theta_{\omega}, l_{\omega}\right)$ be the random vector sampled from mark space $M$ with density function:

$$
g(\omega)=\frac{1}{l_{\max }-l_{\min }} \times \frac{1}{\theta_{\max }-\theta_{\min }}
$$

Then the new segment $w$ is given by:

$$
w(\omega)=\left[x\left(x_{e}, \theta_{\omega}, l_{\omega}\right) y\left(y_{e}, \theta_{\omega}, l_{\omega}\right) \theta_{\omega} l_{\omega}\right]^{T}
$$

and the configuration changes to:

$$
\mathbf{w}^{\prime}=\mathcal{T}(\mathbf{w}, \omega)=[\mathbf{w} w(\omega)]^{T}
$$

Let $P_{C, \text { birth }}$ and $P_{C, \text { death }}$ be the probabilities of choosing the birth and death of connected segments respectively. Then $P_{C, b i r t h} /\left(2 n_{c 0}+n_{c 1}\right)$ is the probability 

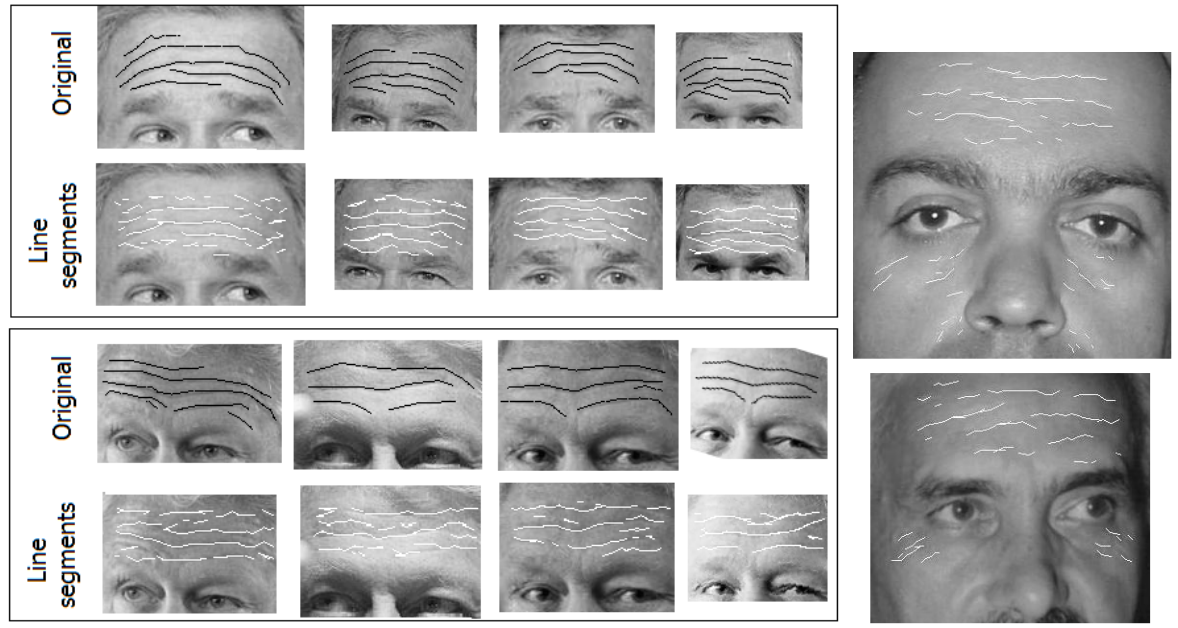

Fig. 4. Detection of wrinkles as line seg-

Fig. 5. Detection of wrinments for two subjects

kles around eyes

of the selection of a particular free end point $\left(x_{e}, y_{e}\right)$ for the birth of a singly connected segment, and $P_{C \text {,death }} / n_{c 1}$ is the probability of the reverse jump of death (deletion) of a connected segment. The Jacobian of bijective transformation, in case of singly connected segments, is equal to one (See Appendix I for derivation). Then the acceptance ratio for this birth move is as follows:

$$
R=\frac{2 n_{c 0}+n_{c 1}}{P_{C, \text { birth }}} \times \frac{P_{C, \text { death }}}{n_{c 1}} \times\left(l_{\max }-l_{\min }\right)\left(\theta_{\max }-\theta_{\min }\right) \times \frac{f\left(\mathbf{w}^{\prime}\right)}{f(\mathbf{w})} .
$$

where the ratio $\frac{f\left(\mathbf{w}^{\prime}\right)}{f(\mathbf{w})}$ is given as:

$$
\frac{f\left(\mathbf{w}^{\prime}\right)}{f(\mathbf{w})}=\frac{f(\mathbf{w} \cup w)}{f(\mathbf{w})}=\beta \exp \left(\alpha \sum_{d \in w}\left(d-d_{\text {min }}\right)^{2}-\sum_{\substack{w_{i} \in \mathbf{w} \\ w_{i} \sim}} q\left(w_{i}, w\right)\right) .
$$

In the case of the birth of a doubly connected segment, the parameter vector $\omega$ for the new line segment $w$ is simply a function of the neighboring two segments and is not sampled randomly. In this case $g(\omega)=1$ and $w=\omega$ which reduces the Jacobian of the bijective transformation to one. Then the acceptance ratio for the birth of a doubly connected segment is given as:

$$
R=\frac{2 n_{c 0}+n_{c 1}}{P_{C, \text { birth }}} \times \frac{P_{C, \text { death }}}{n_{c 2}} \times \frac{f\left(\mathbf{w}^{\prime}\right)}{f(\mathbf{w})} .
$$

The acceptance ratios for the death of connected segments are simply the inverse of the acceptance ratios $1 / R$ for the birth of these segments. 


\subsection{Seed Segments}

The connected segments connect to pre-existing segments with free end points. This makes the placement of the first few free segments critical in the evolution of the Markov Chain in the RJMCMC algorithm. For faster convergence towards the minimum, we propose a favorable initial state by introducing 'seed' segments. The image sites with the highest filter responses are selected while keeping a minimum distance $r_{\text {seed }}$ among them. Seed segments are then placed on these sites in the direction perpendicular to the highest intensity gradient. This results in the placement of seed segments uniformly with inter-seed distance $r_{\text {seed }}$. Figure 3 includes an example of seed segments. Let a seed segment be given as $w(\theta)=\left[x_{\text {seed }} y_{\text {seed }} l_{\text {seed }} \theta\right]^{T}$ and the corresponding set of pixels be $D(w(\theta))$. Then the optimal direction of the seed segment $\theta^{*}$ is found as follows.

$$
\theta^{*}=\arg \max _{\theta \in\left[\theta_{\min }, \theta_{\max }\right]} \sum_{d \in D(w(\theta))} d .
$$

\section{Experiments and Discussion}

For assessing the performance of the detection algorithm on uncontrolled image settings, images were selected from the Internet and the FG-NET database [11. Aging results in wrinkles at several regions of a face i.e. forehead, eye, mouth and nose corners as well as at sagging contours of the skin. For this work, we restricted to the detection of wrinkles on forehead for two reasons (a) generating the ground truth by hand-drawing of wrinkles on the foreheads was easier and (b) wrinkles were more obvious on the forehead in most of the images. Nevertheless, with slight change of parameters in prior model, the wrinkles in other parts of the face can also be detected. For example Figure 5 shows results of detection wrinkles around eye area for two subjects. For each image, the forehead was hand cropped to a rectangle and input to the detection algorithm. It should be mentioned here that the detection of forehead area was not considered under the scope of this work and user-provided cropping was considered sufficient. In total we used 36 images with hand-drawn wrinkles as the ground truth. Where most of the wrinkles were obvious, some were less apparent and posed difficulty even for hand-drawing. The image size varied from $86 \times 37$ as minimum to 290 x 110 as maximum. Resizing images was not considered as we wanted to assess the performance of the detection algorithm on images of different sizes and resolutions.

Evaluation Setup. Since localization of wrinkles is a new application in imagebased analysis of aging skin, we propose an evaluation setup to assess the performance of detection algorithms as well. The terms 'detected', 'original' and 'well-localized' wrinkles are used. The detected wrinkles are the output of the algorithm. The original wrinkles are those hand-drawn by a user and the welllocalized wrinkles are wrinkles detected at correct locations. A margin of $m=3$ pixels is allowed in localization i.e. a detected wrinkle is considered well-localized 

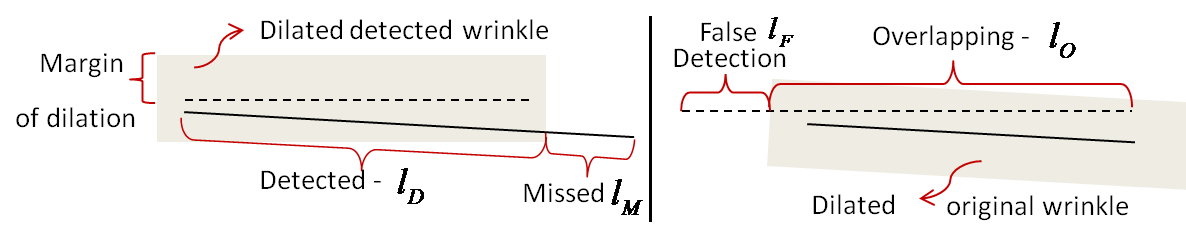

Fig. 6. Evaluation Setup, (left) Calculation for $r_{\text {detect }}$, (right) Calculation for $r_{\text {false }}$

if it is within the distance of $m$ pixels from the hand drawn wrinkle. This way the margin accounts for small inaccuracies in the hand-drawing of wrinkles or small displacements in maximum gradient locations. Morphological dilation with the margin $m$ is used to define the overlap area. A detected wrinkle is considered well-localized if it lies in the overlap area. Let $n_{W}$ and $n_{D}$ be the total number of hand drawn and detected wrinkles respectively. We propose the following ratios for evaluation. Figure 6 shows the detailed procedure for determining the ratios.

1. Detection Ratio $\left(\mathbf{r}_{\text {detect }}\right)$ : The ratio of the total length of original wrinkles within the overlap region of detected wrinkles to the total length of the original wrinkles.

$$
r_{\text {detect }}=\frac{\sum_{n_{W}} l_{\text {overlap }}}{\sum_{n_{W}}\left(l_{\text {overlap }}+l_{\text {miss }}\right)}
$$

2. False Alarm Ratio $\left(\mathbf{r}_{\text {false }}\right)$ : The ratio of the total length of falsely detected wrinkles to the background area with no wrinkles $(\nu(S)$ represents the measure on image space).

$$
r_{\text {false }}=\frac{\sum_{n_{D}} l_{\text {false }}}{\nu(S)-\sum_{n_{W}} l_{\text {original }}}
$$

3. Miss Ratio $\left(\mathbf{r}_{\text {miss }}\right)$ : The ratio of the total length of missed original wrinkles to the total length of original wrinkles.

$$
r_{\text {miss }}=\frac{\sum_{n_{W}} l_{\text {miss }}}{\sum_{n_{W}}\left(l_{\text {overlap }}+l_{\text {miss }}\right)} \text { where } r_{\text {miss }}=1-r_{\text {detect }}
$$

The cropped forehead images were input to the RJMCMC algorithm. It was observed that $2 \times 10^{4}$ iterations were sufficient for the Markov Chain, with the selected cooling schedule, to become stable with almost equal number of birth and death moves. The probabilities $P_{C \text {,birth }}$ and $P_{C \text {,death }}$ were set equal to 0.5 each. The seed parameters $\left\{r_{\text {seed }}, l_{\text {seed }}\right\}$ were fixed at 9 and 4 pixels respectively. However, a better approach in future work would be to select the two parameters based on image resolution. The parameters $\gamma$ and $\alpha$ were selected heuristically. A large value of $\gamma, 150$ in this case, suffices to penalize overlapping or congested segments. The parameter $\alpha$ favors the birth of new segments. Figure 7 (b) shows a plot of results with $\alpha$ varying over a scale of $10^{6}$ for an image (Note that the x-axis has logarithmic scale). The number of segments increases with $\alpha$ increasing both 

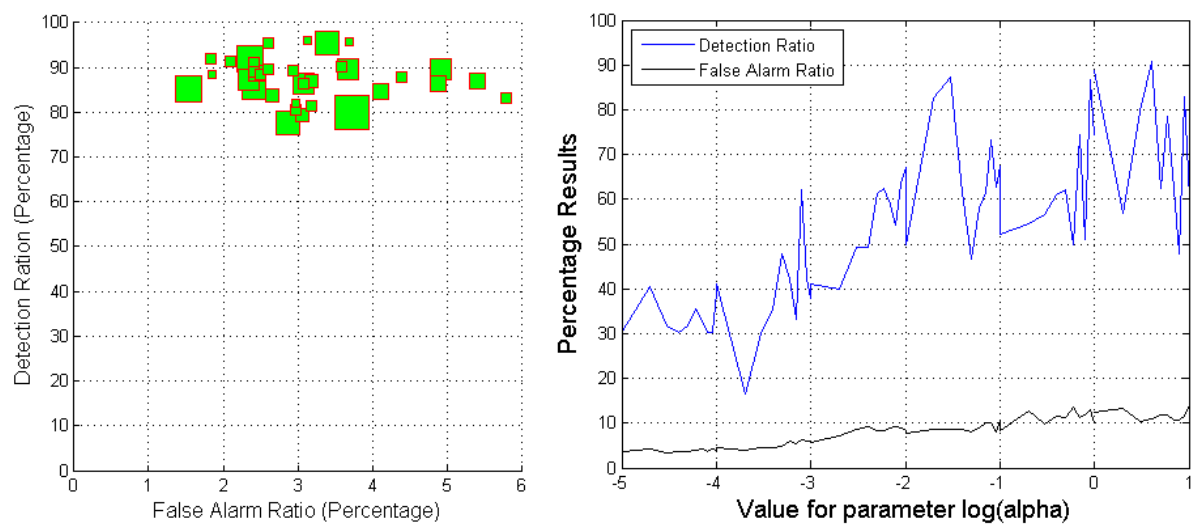

Fig. 7. (a) Detection and False alarm ratios for 36 images of different sizes (b) Effect of value $\alpha$ on detection and false alarm ratios
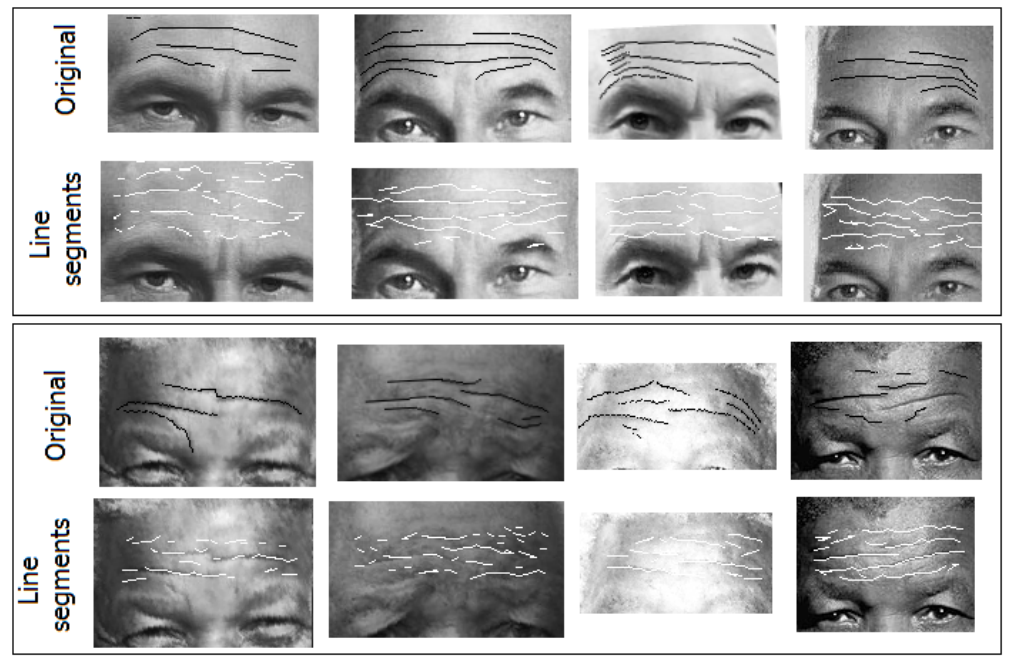

Fig. 8. Detection of wrinkles as line segments for two subjects

detection and false alarm ratios. The optimal values for $\alpha$ were in the range of 0.001-0.03 for smaller images and in the range of $0.00008-0.0005$ for larger images. An unsupervised estimation technique for the parameters, however, is required. The dynamics of the Markov Chain were similar for every image where the total number of segments increased sharply in the beginning and then became stable with equal proportion of birth and death moves. Figure 3 shows the different steps in the algorithm. The increased number of line segments from the initial state of seed segments can be seen after 5000 iterations. Figure 7(a) shows the ROC points for the images. Each plot point is represented by a square whose 


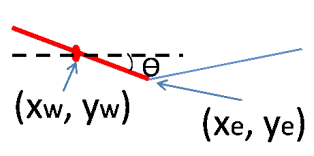

(a)

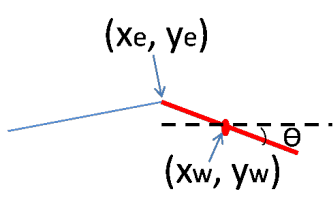

(b)

Fig. 9. Calculation for center coordinates of singly connected segment

size is proportional to the size of the image. Most of the images have detection rates greater than $80 \%$ and false alarm rates around $2.5 \%$ on average. It can be obsereved that the results are independent of the image size. The images on the right side of the plot have relatively lower detection and higher false alarm rates. These are the cases where the algorithm performed relatively poorly because of three reasons (a) some of the wrinkles were not obvious and had lower intensity gradients and, as a result, data likelihood energy values (b) wrinkles were mixed with other skin irregularities and (c) the subject had relatively discontinuous wrinkle curves. Overall, the detection rates were better in subjects with deeper and continuous wrinkles.

\section{Conclusion and Future Work}

In this paper we presented a novel modeling technique for wrinkles based on spatial marked point processes. The model can allow the incorporation of wrinkles, otherwise difficult features to capture, explicitly in future applications. This work will be extended to include estimation of model parameters based on image resolution and representation of wrinkles as curve patterns. The detected wrinkles as spatial curve patterns can be incorporated in biometric applications. Finally, the presented detection algorithm can enable the use of a large set of images with baseline wrinkles for that purpose.

\section{Appendix I}

Let the newly connected segment $w$ be given as:

$$
w=\left[x_{w}\left(x_{e}, \theta_{\omega}, l_{\omega}\right) y_{w}\left(y_{e}, \theta_{\omega}, l_{\omega}\right) \theta_{\omega} l_{\omega}\right]^{T}=\mathcal{T}(\mathbf{w}, \omega)
$$

where $\theta_{\omega} \in\left[\theta_{\min }, \theta_{\max }\right] \subseteq\left[-\frac{\pi}{2}, \frac{\pi}{2}\right]$. From Figure $9(\mathrm{a})$, when the new segment is connected to the left end point, the relationship between $\left(x_{w}, y_{w}\right)$ and $\left(x_{e}, y_{e}\right)$ can be written as:

$$
\begin{aligned}
& x_{w}\left(x_{e}, \theta_{\omega}, l_{\omega}\right)=x_{e}-\frac{l_{\omega}}{2} \cos \left(\theta_{\omega}\right) . \\
& y_{w}\left(y_{e}, \theta_{\omega}, l_{\omega}\right)=y_{e}-\frac{l_{\omega}}{2} \sin \left(\theta_{\omega}\right) .
\end{aligned}
$$


And from Figure 9(b), when the new segment is connected to the right end point, the relationship between $\left(x_{w}, y_{w}\right)$ and $\left(x_{e}, y_{e}\right)$ can be written as:

$$
\begin{aligned}
& x_{w}\left(x_{e}, \theta_{\omega}, l_{\omega}\right)=x_{e}+\frac{l_{\omega}}{2} \cos \left(\theta_{\omega}\right) . \\
& y_{w}\left(y_{e}, \theta_{\omega}, l_{\omega}\right)=y_{e}+\frac{l_{\omega}}{2} \sin \left(\theta_{\omega}\right) .
\end{aligned}
$$

Then the Jacobian factor of bijective tranformation is given as:

$$
\begin{aligned}
& \left|\frac{\partial \mathcal{T}(\mathbf{w}, \omega)}{\partial(\mathbf{w}, \omega)}\right|=\left|\frac{\partial w}{\partial\left(x_{e}, y_{e}, \theta_{\omega}, l_{\omega}\right)}\right| \\
& =\left|\begin{array}{llll}
\frac{\partial x_{w}}{\partial x_{e}} & \frac{\partial y_{w}}{\partial x_{e}} & \frac{\partial \theta_{\omega}}{\partial x_{e}} & \frac{\partial l_{\omega}}{\partial x_{e}} \\
\frac{\partial x_{w}}{\partial y_{e}} & \frac{\partial y_{w}}{\partial y_{e}} & \frac{\partial \theta_{\omega}}{\partial y_{e}} & \frac{\partial l_{\omega}}{\partial y_{e}} \\
\frac{\partial x_{w}}{\partial \theta_{\omega}} & \frac{\partial y_{w}}{\partial \theta_{\omega}} & \frac{\partial \theta_{\omega}}{\partial \theta_{\omega}} & \frac{\partial l_{\omega}}{\partial \theta_{\omega}} \\
\frac{\partial x_{w}}{\partial l_{\omega}} & \frac{\partial y_{w}}{\partial l_{\omega}} & \frac{\partial \theta_{\omega}}{\partial l_{\omega}} & \frac{\partial l_{\omega}}{\partial l_{\omega}}
\end{array}\right| \\
& =\left|\begin{array}{cccc}
1 & 0 & 0 & 0 \\
0 & 1 & 0 & 0 \\
\pm \frac{l_{\omega}}{2} \sin \left(\theta_{\omega}\right) & \mp \frac{l_{\omega}}{2} \cos \left(\theta_{\omega}\right) & 1 & 0 \\
\mp \frac{1}{2} \cos \left(\theta_{\omega}\right) & \mp \frac{1}{2} \sin \left(\theta_{\omega}\right) & 0 & 1
\end{array}\right| \\
& =1
\end{aligned}
$$

\section{References}

1. Fu, Y., Guo, G., Huang, T.: Age synthesis and estimation via faces: A survey. PAMI 32, 1955-1976 (2010)

2. Luu, K., Bui, T.D., Suen, C.Y., Ricanek, K.: Combined local and holistic facial features for age-determination. In: ICCARV, pp. 900-904 (2010)

3. Suo, J., Min, F., Zhu, S., Shan, S., Chen, X.: A multi-resolution dynamic model for face aging simulation. In: CVPR, pp. 1-8 (2007)

4. Kwon, Y.H., Lobo, N.D.V.: Age classification from facial images. In: CVPR (1999)

5. Cula, G.O., Bargo, P.R., Kollias, N.: Assessing facial wrinkles: automatic detection and quantification, vol. 7161, pp. 71610J-71610J-6 (2009)

6. Cula, O.G., Dana, K.J., Murphy, F.P., Rao, B.K.: Skin texture modeling. IJCV 62, 97-119 (2005)

7. Lieshout, M.N.M.: Markov Point Processes and their Applications. Imperial College Press/World Scientific Publishing, London, Singapore (2000)

8. Stoica, R., Descombes, X., Zerubia, J.: A Gibbs point process for road extraction from remotely sensed images. IJCV 57, 121-136 (2004)

9. Green, P.: Reversible jump MCMC computation and Bayesian model determination. Biometrika 82, 711-732 (1995)

10. Geyer, C.J., Moller, J.: Simulation procedures and likelihood inference for spatial point processes. Scandinavian Journal of Statistics 21, 359-373 (1994)

11. Available: The FG-net aging database, http://www.fgnet.rsunit.com/ 